Artículo original

\title{
Desempeño del sistema de vigilancia colombiano durante la pandemia de COVID-19: evaluación rápida de los primeros $\mathbf{5 0}$ días
}

\author{
Edgar F. Manrique-Hernández¹, José Moreno-Montoya² ${ }^{2}$ Alexandra Hurtado-Ortiz ${ }^{1}$ \\ Franklyn E. Prieto-Alvarado ${ }^{3}$, Álvaro J. Idrovo ${ }^{1}$ \\ ${ }^{1}$ Departamento de Salud Pública, Escuela de Medicina, Universidad Industrial de Santander, \\ Bucaramanga, Colombia \\ ${ }^{2}$ Subdirección de Estudios Clínicos, Fundación Santa Fe de Bogotá, Bogotá D.C., Colombia \\ ${ }^{3}$ Dirección de Vigilancia y Análisis del Riesgo en Salud Pública, Instituto Nacional de Salud, \\ Bogotá, D.C., Colombia
}

Introducción. La pandemia de COVID es un desafío para la vigilancia en salud pública y una oportunidad para evaluar sus fortalezas y debilidades en aras de mejorar la respuesta. Objetivo. Evaluar el desempeño del sistema de vigilancia en salud pública colombiano durante los primeros 50 días de la pandemia de COVID-19 en el país.

Materiales y métodos. Se analizaron los datos publicados entre el 6 de marzo y el 24 de abril de 2020 por el Instituto Nacional de Salud y la Organización Mundial de Salud (OMS). Se consideraron en la evaluación: i) la calidad de los datos según la ley de Benford y ii) la oportunidad de la información, medida como la diferencia en fechas entre los datos generados en el Instituto Nacional de Salud y los recogidos en el informe situacional de la OMS. La variabilidad en el cumplimiento de la ley de Benford se evaluó con los valores de p en las pruebas de razón del logaritmo de la verosimilitud, ji al cuadrado o exacta de Moreno. Resultados. Hasta el 24 de abril hubo 4.881 casos de COVID-19 en Colombia. En la mayoría de los primeros 50 días se cumplió la ley de Benford, excepto en los primeros días de la epidemia. La diferencia entre los informes del Instituto Nacional de Salud y la OMS ha dependido, en gran medida, de la diferencia en los horarios de cierre de la información. Conclusión. En general, el sistema de vigilancia en salud pública colombiano cumplió con la ley de Benford, lo cual sugiere que hubo calidad en los datos. En futuros estudios que comparen el desempeño de los departamentos y distritos se podrá mejorar el diagnóstico de la vigilancia en salud pública del país.

Recibido: 08/05/2020

Aceptado: 21/05/2020

Publicado: $21 / 05 / 2020$

Citación:

Manrique-Hernández EF, Moreno-Montoya J, Hurtado-Ortiz A, Prieto-Alvarado FE, Idrovo AJ. Desempeño del sistema de vigilancia colombiano durante la pandemia de COVID-19: evaluación rápida de los primeros 50 días. Biomédica. 2020;40(Supl.2):96-103.

https://doi.org/10.7705/biomedica.5582

\section{Correspondencia:}

Álvaro Javier Idrovo, Departamento de Salud Pública, Escuela de Medicina, Universidad Industrial de Santander, Carrera 32 № 29-31, Bucaramanga, Colombia

Teléfono: (577) 634 4000, extensión 3195

idrovoaj@yahoo.com.mx

\section{Contribución de los autores:}

Edgar F. Manrique-Hernández, José MorenoMontoya y Alexandra Hurtado-Ortiz: participación en todas las etapas del estudio

Álvaro J. Idrovo: análisis de los datos

Todos los autores participaron en la discusión de resultados y en la redacción del manuscrito.

Financiación:

El estudio no tuvo financiamiento.

\section{Conflicto de intereses:}

Franklyn E. Prieto-Alvarado es el director nacional de Vigilancia y Análisis del Riesgo en Salud Pública del Instituto Nacional de Salud, responsable de dirigir el proceso de vigilancia en salud pública durante la pandemia.
Palabras clave: infecciones por coronavirus; epidemias; notificación de enfermedad; monitoreo epidemiológico; distribuciones estadísticas; Colombia.

Performance of the Colombian surveillance system during the COVID-19 pandemic: A rapid evaluation of the first 50 days

Introduction: The COVID pandemic is a challenge for public health surveillance and an opportunity to assess its strengths and weaknesses to improve the response.

Objective: To evaluate the performance of the Colombian public health surveillance system during the first 50 days of the COVID-19 pandemic in the country.

Materials and methods: We analyzed the data published between March 6 and April 24, 2020, by the Instituto Nacional de Salud and the World Health Organization (WHO). We evaluated: i) the quality of the data according to the fulfillment of Benford's law, and ii) the timeliness of the information measured as the difference in dates between the data generated by the Instituto Nacional de Salud and WHO's situational reports. We assessed the fulfillment of Benford's law using the $p$ values of the log-likelihood ratio, the chi square or Moreno's exact tests.

Results: Until April 24 there were 4,881 cases of COVID-19 in Colombia. During most of the first 50 days of the pandemic, Benford's law was fulfilled except the first days of the epidemic. The difference between Instituto Nacional de Salud and WHO reports largely depends on the different reporting times.

Conclusion: In general, the Colombian public health surveillance system fulfilled Benford's law suggesting that there was quality in the data. Future studies comparing the performance of the departments and districts will improve the diagnosis of the Colombian surveillance system.

Keywords: Coronavirus infections; epidemics; disease notification; epidemiological monitoring; statistical distributions; Colombia. 
La vigilancia en salud pública es una herramienta útil para la monitorización de enfermedades, particularmente las infecciosas, y se originó en el puerto marítimo de Venecia, Italia, en 1348, cuando tres guardianes de salud pública tenían como función detectar e impedir el desembarco de personas con peste negra. Este tipo de iniciativas dieron origen a estrategias preventivas como la cuarentena, la detección temprana de casos y el aislamiento (1). Con el paso de los años, dicha vigilancia se ha ido refinando hasta convertirse en un elemento esencial para el manejo sanitario y la seguridad en cualquier país, especialmente en cuanto a las enfermedades infecciosas con potencial pandémico $(2,3)$.

Según los Centers for Disease Control and Prevention (CDC) de los Estados Unidos, la vigilancia en salud pública consiste en la "recolección continua y sistemática, el análisis, la interpretación y la difusión de datos sobre un evento relacionado con la salud para su uso en acciones de salud pública encaminadas a reducir la morbilidad y la mortalidad y mejorar salud" (4). Si bien existen otras definiciones, todas con elementos comunes, la vigilancia en salud pública es la herramienta fundamental para el control de las epidemias, especialmente en lo tocante a los tratamientos o las vacunas para su prevención $(5,6)$, como en el caso de la pandemia de COVID-19.

En Colombia, la vigilancia en salud pública no ha sido ajena a los desarrollos técnico-científicos y ha seguido fundamentalmente la estrategia de formación de epidemiólogos de campo del CDC (7). Un hito en la historia de la epidemiología de campo en Colombia ocurrió en 1993, cuando se inició la consolidación del grupo del Instituto Nacional de Salud encargado del manejo y el estudio de epidemias bajo parámetros modernos.

A partir de entonces, han sido innumerables los casos de manejo de conglomerados y epidemias que pueden considerarse como éxitos de la epidemiología de campo colombiana (8). Es el caso del manejo de pandemias como la de la influenza $A\left(H_{1} N_{1}\right)$ en el 2009, que permitió hacer una evaluación de la percepción de las competencias de los epidemiólogos y profesionales de salud pública para manejar este tipo de emergencias sanitarias (9) y contrastarla con los análisis objetivos del desempeño de la vigilancia en salud pública de otros países (10). En ese momento pudieron detectarse, asimismo, algunas deficiencias que han tenido diversos cursos de solución con el paso del tiempo.

En ese sentido, el presente trabajo tuvo como objetivo evaluar el desempeño del sistema de vigilancia en salud pública colombiano durante los primeros 50 días de la pandemia de COVID-19 en el país.

\section{Materiales y métodos}

Se analizaron los datos oficiales del sistema colombiano disponibles en la página web del Instituto Nacional de Salud (https://www.ins.gov.co/Noticias/ Paginas/Coronavirus.aspx) y los informes situacionales de la Organización Mundial de la Salud (OMS) (https://www.who.int/emergencies/diseases/novelcoronavirus-2019/situation-reports/). Si bien el sistema de vigilancia en salud pública está activo todo el tiempo, se incluyeron en este análisis los datos a partir del día en que fue diagnosticado el primer caso de COVID-19, el cual se reportó en Bogotá el 6 de marzo de 2020.

Se entiende que la evaluación cobija todos los procesos involucrados en las labores de los epidemiólogos de campo en los equipos de respuesta inmediata, 
así como el procesamiento de muestras en el laboratorio y la generación de los reportes situacionales que se divulgan a nivel nacional e internacional.

Se consideraron dos indicadores: i) la calidad de los datos según el cumplimiento de la ley de Benford, y ii) la oportunidad de la información. Este último indicador se consideró en atención a las necesidades internacionales o fronterizas de vigilancia de los fenómenos migratorios en el curso de una pandemia.

\section{La ley de Benford}

Esta ley matemática, también denominada "ley de los primeros dígitos", "ley de Newcomb-Benford" o "ley de los números anómalos" (11), señala que en un conjunto de números, aquellos que empiezan con el número 1 son más frecuentes que los que empiezan con otros dígitos, siguiendo un orden decreciente que se puede expresar como $P(d)=\log [1+(1=d)] d=1,2, \ldots, 9$, donde una serie de números $\mathrm{P}(\mathrm{d})$ corresponde a la probabilidad de que un dígito sea el número inicial $(12,13)$.

Este método matemático ha sido particularmente útil y exitoso en la detección de datos fraudulentos $(14,15)$. En el campo de la vigilancia en salud pública, se usó por primera vez durante la pandemia de influenza $A\left(H_{1} N_{1}\right)$ y, después, en las epidemias de dengue en Paraguay (16), de Zika en América (17) y de COVID-19 en China (18).

La oportunidad de la información se evaluó calculando la diferencia entre el tiempo en que se informaba el número de casos y fallecidos de Colombia en el portal del Instituto Nacional de Salud y el tiempo en que aparecía dicha información en el portal de la OMS. Dada la importancia de contar con información integral y oportuna a nivel mundial para definir las acciones multinacionales, este indicador puede entenderse como una evaluación de la magnitud del "rezago temporal".

\section{Métodos estadísticos}

Para evaluar el nivel de cumplimiento de la distribución descrita por la ley de Benford en los datos observados (casos diarios acumulados), se usaron las pruebas de razón del logaritmo de la verosimilitud y de ji al cuadrado mediante la macro digdis desarrollada por Ben Jann (ETH, Zurich). Para verificar el cumplimiento de la ley de Benford en los datos de las muestras pequeñas durante los primeros días de reporte, se usó la prueba exacta de Moreno (19), ya que resultó tener un mejor desempeño que la prueba de Kuiper (20). Dichos análisis se hicieron con el programa estadístico Stata $14^{\mathrm{TM}}$ (Stata Corporation, USA) o el programa R.

\section{Resultados}

En el cuadro 1 se encuentran los resultados del análisis del cumplimiento de la ley de los primeros dígitos en el número de casos acumulados día a día desde el 6 de marzo hasta el 24 de abril de 2020.

Dado que las pruebas estadísticas para evaluar el cumplimiento de la ley de Benford son pruebas de bondad de ajuste, los valores de $p$ más pequeños sugieren que esta no se cumplió. Debido a que se contaba con pocas observaciones en los primeros días, para estos datos se usó exclusivamente la prueba exacta de Moreno, cuyos resultados sugieren un buen desempeño el primer día; después disminuyó la calidad de los datos hasta que entre los días 3 y 11 de la vigilancia (8 a 16 de marzo) no se cumplió la ley de Benford. 
Cuadro 1. Cumplimiento de la ley de Benford de cada uno de los reportes de número de casos de COVID-19 en Colombia (casos acumulados)

\begin{tabular}{|c|c|c|c|c|c|c|c|c|c|c|c|c|c|c|}
\hline \multirow{2}{*}{$\begin{array}{l}\text { Reporte } \\
\text { (n) }\end{array}$} & \multirow{2}{*}{ Fecha } & \multirow{2}{*}{$\begin{array}{c}\text { Casos } \\
\text { acumulados }\end{array}$} & \multicolumn{9}{|c|}{ Primeros dígitos } & \multirow{2}{*}{$\begin{array}{l}\text { Exacta de } \\
\text { Moreno }\end{array}$} & \multirow{2}{*}{$\begin{array}{l}\text { Razón del logaritmo } \\
\text { de la verosimilitud }\end{array}$} & \multirow{2}{*}{$\chi^{2}$} \\
\hline & & & 1 & 2 & 3 & 4 & 5 & 6 & 7 & 8 & 9 & & & \\
\hline 1 & $06 / 03 / 2020$ & 1 & 1 & 0 & 0 & 0 & 0 & 0 & 0 & 0 & 0 & 0,3010 & & \\
\hline 2 & 07/03/2020 & 1 & 2 & 0 & 0 & 0 & 0 & 0 & 0 & 0 & 0 & 0,0906 & & \\
\hline 3 & 08/03/2020 & 1 & 3 & 0 & 0 & 0 & 0 & 0 & 0 & 0 & 0 & 0,0272 & & \\
\hline 4 & 09/03/2020 & 3 & 3 & 0 & 1 & 0 & 0 & 0 & 0 & 0 & 0 & 0,0136 & & \\
\hline 5 & $10 / 03 / 2020$ & 3 & 3 & 0 & 2 & 0 & 0 & 0 & 0 & 0 & 0 & 0,0042 & & \\
\hline 6 & $11 / 03 / 2020$ & 9 & 3 & 0 & 2 & 0 & 0 & 0 & 0 & 0 & 1 & 0,0011 & & \\
\hline 7 & 12/03/2020 & 9 & 3 & 0 & 2 & 0 & 0 & 0 & 0 & 0 & 2 & 0,0001 & & \\
\hline 8 & $13 / 03 / 2020$ & 16 & 4 & 0 & 2 & 0 & 0 & 0 & 0 & 0 & 2 & 0,0001 & 0,0920 & 0,0936 \\
\hline 9 & 14/03/2020 & 24 & 4 & 1 & 2 & 0 & 0 & 0 & 0 & 0 & 2 & 0,0001 & 0,2121 & 0,2129 \\
\hline 10 & 15/03/2020 & 45 & 4 & 1 & 2 & 1 & 0 & 0 & 0 & 0 & 2 & 0,0001 & 0,3434 & 0,3544 \\
\hline 11 & 16/03/2020 & 57 & 4 & 1 & 2 & 1 & 1 & 0 & 0 & 0 & 2 & 0,0001 & 0,4986 & 0,5055 \\
\hline 12 & 17/03/2020 & 75 & 4 & 1 & 2 & 1 & 1 & 0 & 1 & 0 & 2 & & 0,6284 & 0,6243 \\
\hline 13 & 18/03/2020 & 102 & 5 & 1 & 2 & 1 & 1 & 0 & 1 & 0 & 2 & & 0,5890 & 0,6356 \\
\hline 14 & 19/03/2020 & 128 & 6 & 1 & 2 & 1 & 1 & 0 & 1 & 0 & 2 & & 0,5232 & 0,6081 \\
\hline 15 & $20 / 03 / 2020$ & 158 & 7 & 1 & 2 & 1 & 1 & 0 & 1 & 0 & 2 & & 0,4413 & 0,5505 \\
\hline 16 & $21 / 03 / 2020$ & 210 & 7 & 2 & 2 & 1 & 1 & 0 & 1 & 0 & 2 & & 0,5710 & 0,6953 \\
\hline 17 & $22 / 03 / 2020$ & 235 & 7 & 3 & 2 & 1 & 1 & 0 & 1 & 0 & 2 & & 0,6058 & 0,7607 \\
\hline 18 & $23 / 03 / 2020$ & 306 & 7 & 3 & 3 & 1 & 1 & 0 & 1 & 0 & 2 & & 0,5775 & 0,7604 \\
\hline 19 & $24 / 03 / 2020$ & 378 & 7 & 3 & 4 & 1 & 1 & 0 & 1 & 0 & 2 & & 0,4891 & 0,6793 \\
\hline 20 & $25 / 03 / 2020$ & 470 & 7 & 3 & 4 & 2 & 1 & 0 & 1 & 0 & 2 & & 0,5422 & 0,7548 \\
\hline 21 & 26/03/2020 & 491 & 7 & 3 & 4 & 3 & 1 & 0 & 1 & 0 & 2 & & 0,4947 & 0,7261 \\
\hline 22 & $27 / 03 / 2020$ & 539 & 7 & 3 & 4 & 3 & 2 & 0 & 1 & 0 & 2 & & 0,5255 & 0,7768 \\
\hline 23 & 28/03/2020 & 608 & 7 & 3 & 4 & 3 & 2 & 1 & 1 & 0 & 2 & & 0,8306 & 0,9140 \\
\hline 24 & 29/03/2020 & 702 & 7 & 3 & 4 & 3 & 2 & 1 & 2 & 0 & 2 & & 0,8156 & 0,9097 \\
\hline 25 & $30 / 03 / 2020$ & 798 & 7 & 3 & 4 & 3 & 2 & 1 & 3 & 0 & 2 & & 0,6969 & 0,7875 \\
\hline 26 & $31 / 03 / 2020$ & 906 & 7 & 3 & 4 & 3 & 2 & 1 & 3 & 0 & 3 & & 0,5296 & 0,5611 \\
\hline 27 & 01/04/2020 & 1.065 & 8 & 3 & 4 & 3 & 2 & 1 & 3 & 0 & 3 & & 0,5430 & 0,6018 \\
\hline 28 & 02/04/2020 & 1.161 & 9 & 3 & 4 & 3 & 2 & 1 & 3 & 0 & 3 & & 0,5374 & 0,6214 \\
\hline 29 & 03/04/2020 & 1.267 & 10 & 3 & 4 & 3 & 2 & 1 & 3 & 0 & 3 & & 0,5157 & 0,6219 \\
\hline 30 & 04/04/2020 & 1.406 & 11 & 3 & 4 & 3 & 2 & 1 & 3 & 0 & 3 & & 0,4807 & 0,6050 \\
\hline 31 & 05/04/2020 & 1.485 & 12 & 3 & 4 & 3 & 2 & 1 & 3 & 0 & 3 & & 0,4354 & 0,5727 \\
\hline 32 & 06/04/2020 & 1.579 & 13 & 3 & 4 & 3 & 2 & 1 & 3 & 0 & 3 & & 0,3833 & 0,5273 \\
\hline 33 & 07/04/2020 & 1.780 & 14 & 3 & 4 & 3 & 2 & 1 & 3 & 0 & 3 & & 0,3277 & 0,4714 \\
\hline 34 & 08/04/2020 & 2.054 & 14 & 4 & 4 & 3 & 2 & 1 & 3 & 0 & 3 & & 0,4214 & 0,5809 \\
\hline 35 & 09/04/2020 & 2.223 & 14 & 5 & 4 & 3 & 2 & 1 & 3 & 0 & 3 & & 0,4816 & 0,6589 \\
\hline 36 & $10 / 04 / 2020$ & 2.473 & 14 & 6 & 4 & 3 & 2 & 1 & 3 & 0 & 3 & & 0,5087 & 0,7036 \\
\hline 37 & $11 / 04 / 2020$ & 2.709 & 14 & 7 & 4 & 3 & 2 & 1 & 3 & 0 & 3 & & 0,5065 & 0,7176 \\
\hline 38 & $12 / 04 / 2020$ & 2.776 & 14 & 8 & 4 & 3 & 2 & 1 & 3 & 0 & 3 & & 0,4801 & 0,7036 \\
\hline 39 & 13/04/2020 & 2.852 & 14 & 9 & 4 & 3 & 2 & 1 & 3 & 0 & 3 & & 0,4348 & 0,6634 \\
\hline 40 & $14 / 04 / 2020$ & 2.979 & 14 & 10 & 4 & 3 & 2 & 1 & 3 & 0 & 3 & & 0,3763 & 0,5990 \\
\hline 41 & $15 / 04 / 2020$ & 3.105 & 14 & 10 & 5 & 3 & 2 & 1 & 3 & 0 & 3 & & 0,3981 & 0,6410 \\
\hline 42 & $16 / 04 / 2020$ & 3.233 & 14 & 10 & 6 & 3 & 2 & 1 & 3 & 0 & 3 & & 0,3875 & 0,6437 \\
\hline 43 & $17 / 04 / 2020$ & 3.439 & 14 & 10 & 7 & 3 & 2 & 1 & 3 & 0 & 3 & & 0,3518 & 0,6098 \\
\hline 44 & $18 / 04 / 2020$ & 3.621 & 14 & 10 & 8 & 3 & 2 & 1 & 3 & 0 & 3 & & 0,2995 & 0,5429 \\
\hline 45 & $19 / 04 / 2020$ & 3.792 & 14 & 10 & 9 & 3 & 2 & 1 & 3 & 0 & 3 & & 0,2394 & 0,4499 \\
\hline 46 & $20 / 04 / 2020$ & 3.977 & 14 & 10 & 10 & 3 & 2 & 1 & 3 & 0 & 3 & & 0,1798 & 0,3431 \\
\hline 47 & $21 / 04 / 2020$ & 4.149 & 14 & 10 & 10 & 4 & 2 & 1 & 3 & 0 & 3 & & 0,2078 & 0,3993 \\
\hline 48 & $22 / 04 / 2020$ & 4.356 & 14 & 10 & 10 & 5 & 2 & 1 & 3 & 0 & 3 & & 0,2107 & 0,4200 \\
\hline 49 & $23 / 04 / 2020$ & 4.561 & 14 & 10 & 10 & 6 & 2 & 1 & 3 & 0 & 3 & & 0,1931 & 0,4038 \\
\hline 50 & 24/04/2020 & 4.881 & 14 & 10 & 10 & 7 & 2 & 1 & 3 & 0 & 3 & & 0,1622 & 0,3554 \\
\hline
\end{tabular}


Para los datos a partir del día 8 de la vigilancia (13 de marzo) la evaluación se hizo con las pruebas de razón del logaritmo de la verosimilitud y de ji al cuadrado, las cuales registraron una mejoría en el cumplimiento de la ley de Benford; los días de mejor desempeño fueron el 28 y el 29 de marzo $(p>0,80)$. Con valores más bajos, aunque estadísticamente satisficieron la ley de Benford, aparecen los días 20, 23 y 24 de abril, con valores de $p$ entre 0,15 y 0,20 . En el cuadro 2 y la figura 1 se presentan en detalle los resultados del último día de vigilancia incluido en este análisis.

En la figura 2 se observan los casos acumulados según lo reportado por el Instituto Nacional de Salud y la OMS para Colombia en las mismas fechas. Como se puede apreciar, la línea azul se encuentra siempre por debajo de la línea negra, lo que equivale a una igualdad en los reportes, por lo que se concluye que hay un claro rezago (de dos días usualmente) en la información publicada por la OMS.

Cuadro 2. Cumplimiento de la ley de Benford en el día 50 de epidemia de COVID-19 en Colombia (casos acumulados)

\begin{tabular}{lcrl}
\hline Primer dígito & Conteo & $\%$ & $\mathbf{p}$ \\
\hline 1 & 14 & 28 & 0,8777 \\
2 & 10 & 20 & 0,7095 \\
3 & 10 & 20 & 0,1295 \\
4 & 7 & 14 & 0,3322 \\
5 & 2 & 4 & 0,4338 \\
6 & 1 & 2 & 0,2592 \\
7 & 3 & 6 & 0,7659 \\
8 & 0 & 0 & 0,1838 \\
9 & 3 & 6 & 0,4987 \\
\hline
\end{tabular}

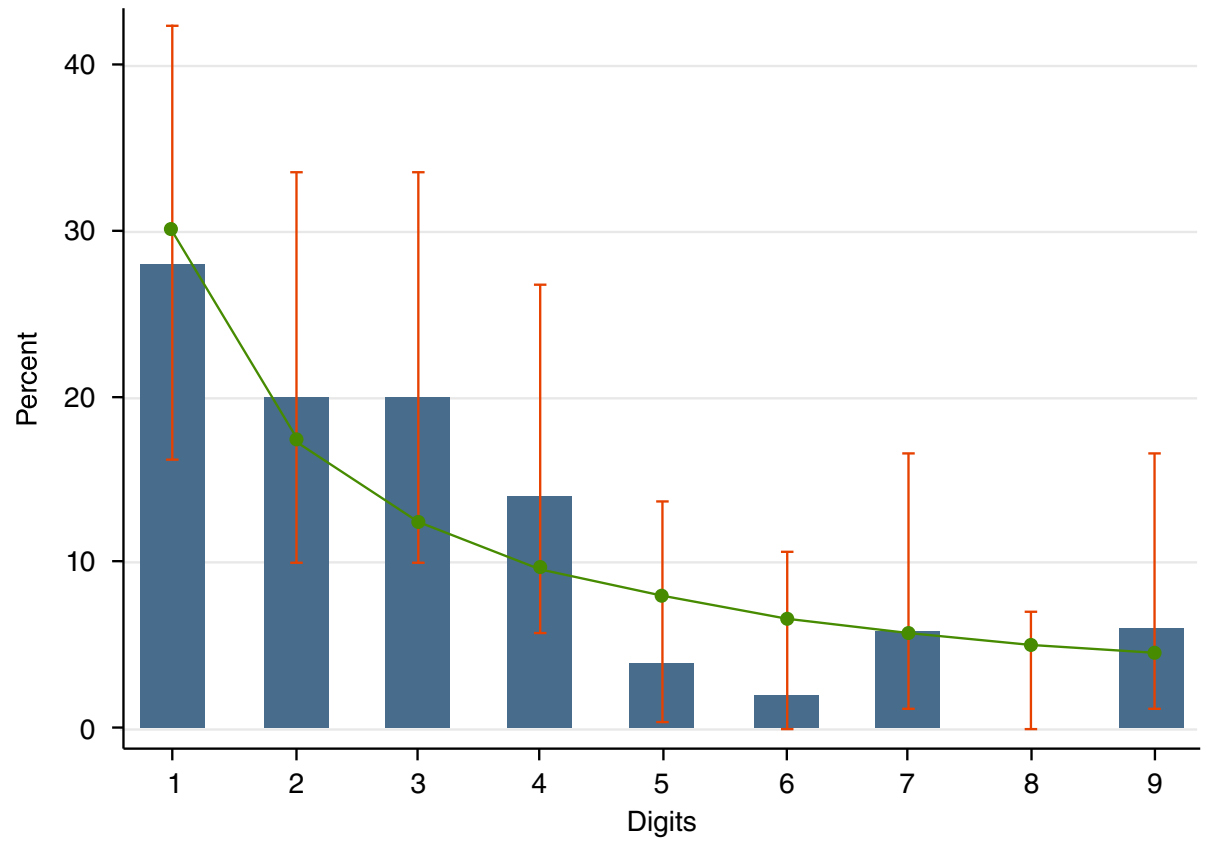

Las columnas corresponden a los datos obtenidos del reporte, con sus intervalos de confianza, y la línea en verde a la distribución teórica de la ley de Benford

Figura 1. Frecuencia de los primeros dígitos en la distribución de la ley de Benford reportados en el último día analizado 


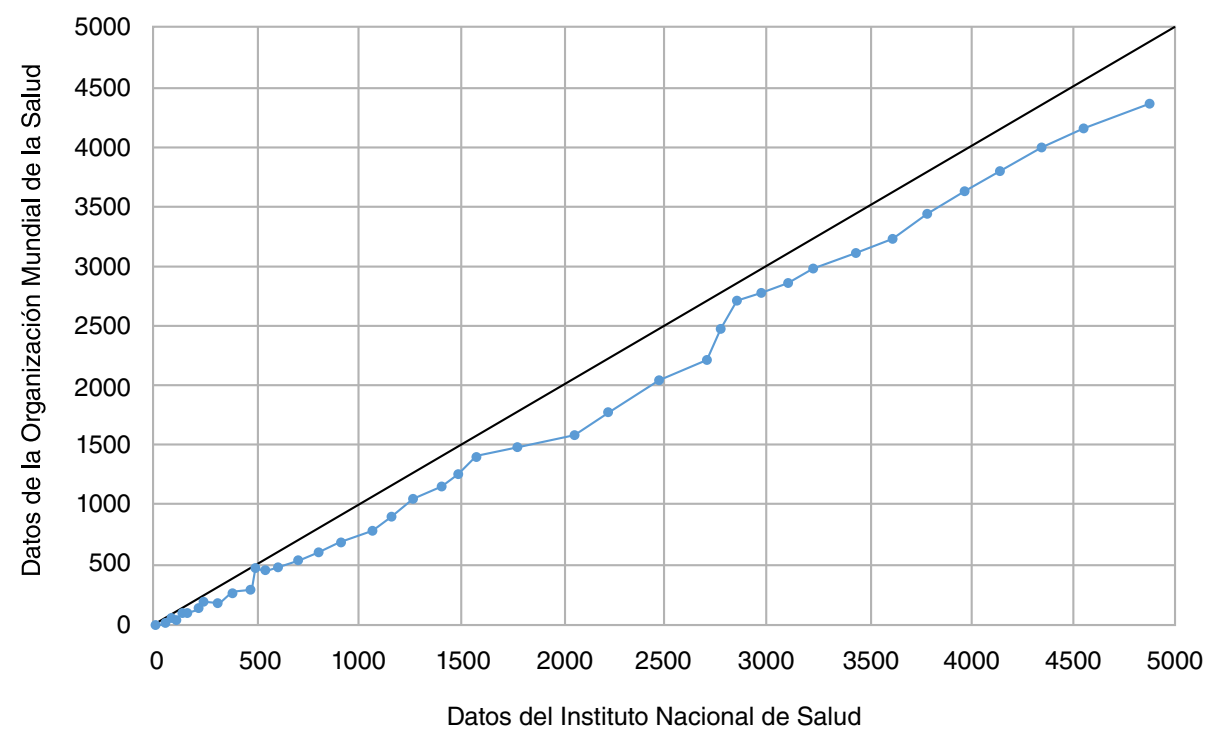

Figura 2. Diferencia en los casos acumulados diarios de COVID-19 reportados en los portales del Instituto Nacional de Salud y la Organización Mundial de la Salud (6 de marzo y el 24 de abril de 2020).

\section{Discusión}

Los hallazgos de estos análisis sugieren que los primeros días de la vigilancia del COVID-19 en territorio colombiano fueron los más difíciles y la calidad de los datos no fue óptima. En los dos primeros días se cumplió la ley de Benford, al no registrarse más que un solo caso; luego, entre el día 3 y el día 11 (8 a 16 de marzo), la ley de los primeros dígitos no se cumplió porque durante tres días hubo un único caso y se pasó directo a tres y de allí a nueve casos de COVID-19. Este es un comportamiento esperable dado que apenas se empezaban a afinar las actividades de la vigilancia en salud pública.

La problemática de los primeros días de epidemia en Colombia se relacionó con las dificultades para garantizar la continuidad de la oferta de la prueba por no disponerse de suficientes reactivos, de equipos para la operación en serie, de infraestructura a nivel regional y local para descentralizar el diagnóstico, y por el proceso particular de la prueba estandarizada por la OMS, entre otros.

En los primeros días también se detectó que se priorizaba la prueba sobre el registro de la vigilancia, situación que se fue ajustando rápidamente. Además, algunos niveles locales asumieron la gobernanza para organizar más estructuradamente en su territorio la notificación, la investigación epidemiológica de campo, el estudio de contactos y la recolección de muestras. Después de esos primeros días, en general, los datos de la vigilancia en salud pública sugieren que se ha cumplido con la ley de Benford, aunque con fluctuaciones. El cumplimiento de la ley de Benford a juzgar por los valores de $p$ de la prueba de razón del logaritmo de la verosimilitud muestra altibajos.

Al poner estos datos en el contexto que brindan los análisis del desempeño de la vigilancia en salud pública durante las epidemias de influenza $A\left(H_{1} N_{1}\right)$, de Zika y ahora de COVID-19 (cuadro 3), se observa que su ejercicio en Colombia se ha consolidado y que el componente basado en el laboratorio (21) ha tenido mejor desempeño cuando los agentes infecciosos son transmitidos de persona a persona por vía respiratoria que cuando estos son 
Cuadro 3. Cumplimiento de la ley de Benford (valores de $p$ en la prueba de razón del logaritmo de la verosimilitud) de la vigilancia en salud pública en Colombia durante las epidemias de influenza $A(\mathrm{H} 1 \mathrm{~N} 1)$, Zika y COVID-19

\begin{tabular}{lccc}
\hline Casos & $\begin{array}{c}\text { Influenza A(H1N1) } \\
\text { 2009 }\end{array}$ & $\begin{array}{c}\text { Virus Zika } \\
\text { 2016 }\end{array}$ & $\begin{array}{c}\text { Virus COVID-19 } \\
\text { 2020* }\end{array}$ \\
\hline Confirmados (semanas) & $0,190(25)$ & $0,068(42)$ & $0,162(50)$ \\
Sospechosos (semanas) & ND & $0,467(41)$ & ND \\
\hline
\end{tabular}

* Incluye solamente los primeros 50 días a partir del diagnóstico del primer caso en Colombia.

transmitidos por insectos vectores. Sin embargo, cabe destacar que el mejor desempeño del sistema se registró con los casos probables de Zika valorados a partir de criterios clínicos y epidemiológicos, y relacionado con un mayor reconocimiento de cuadros sindrómicos específicos, como el de Guillain-Barré, y el de los defectos congénitos relacionados (22).

En cuanto a la oportunidad de la información, se pudo observar un rezago temporal importante entre el Instituto Nacional de Salud y la OMS, que corresponde, en gran medida, a las diferentes horas de cierre de cada informe situacional. La OMS suele hacer su cierre de datos hacia las 03:00 hora de Colombia (10:00, hora de verano de Europa Central), en tanto que el Instituto Nacional de Salud ha variado las horas, siendo más frecuente en las tardes (14:00 a 18:00) de cada día.

En este sentido, es interesante anotar que el Center for Systems Science and Engineering (CSSE) de la Johns Hopkins University organizó un tablero interactivo en internet (https://coronavirus.jhu.edu/) que disminuye notoriamente este rezago temporal, pues recibe directamente de cada país o territorio los datos y va actualizando la información, lo que permite tenerla casi en tiempo real (23). Este tablero ha sido la fuente más confiable en tiempo real de la pandemia alrededor del mundo.

Uno de los avances más importantes del sistema de vigilancia colombiano es la disponibilidad de datos abiertos (https://www.ins.gov.co/Noticias/ Paginas/Coronavirus.aspx), que permiten verificar la oportunidad del sistema en términos del reporte de los casos confirmados, casos que han sido investigados desde la condición probable en términos de la contención.

Los hallazgos aquí descritos no corresponden a una evaluación completa de un sistema de vigilancia en salud pública. Otros elementos como la simplicidad, la flexibilidad, la aceptabilidad, la sensibilidad, la representatividad, la estabilidad y el valor predictivo positivo (24) deben contemplarse para tener una visión integral. Sin embargo, este análisis preliminar de la calidad de los datos y de su oportunidad en etapas iniciales de una epidemia resulta muy útil, pues permite realimentar rápidamente el sistema de vigilancia, con el fin de adoptar medidas correctivas si es el caso.

Dado que en Colombia son muy diversas las capacidades en salud pública y epidemiología, en futuros análisis se podrán evaluar los sistemas de vigilancia en los departamentos y distritos para así determinar acciones a ese nivel que favorezcan el mejoramiento continuo de la vigilancia en salud pública.

\section{Referencias}

1. Declich S, Carter AO. Public health surveillance: Historical origins, methods and evaluation. Bull World Health Organ. 1994;72:285-304.

2. Aldis W. Health security as a public health concept: a critical analysis. Health Pol Plan. 2008;23:369-75. https://doi.org/10.1093/heapol/czn030 
3. Weir L, Mykhalovskiy E. The geopolitics of global public health surveillance in the twenty-first century. In: Bashford A, editors. Medicine at the border. London: Palgrave Macmillan; 2007. p. 240-63.

4. Centers for Disease Control and Prevention. Guidelines for evaluating surveillance systems. MMWR Suppl. 1988;37:1-18.

5. Bedford J, Enria D, Giesecke J, Heymann DL, Ihekweazu C, Kobinger G, et al. COVID-19: Towards controlling of a pandemic. Lancet. 2020;395:1015-8. http://doi.org/10.1016/S0140-6736(20)30673-5

6. Pung R, Chiew CJ, Young BE, Chin S, Chen MIC, Clapham HE, et al. Investigation of three clusters of COVID-19 in Singapore: Implications for surveillance and response measures. Lancet. 2020;395:1039-46. http://doi.org/10.1016/S0140-6736(20)30528-6

7. Idrovo AJ, Eslava JC, Ruiz-Rodríguez M, Rodríguez JM. La otra transición epidemiológica: hitos en el desarrollo de la epidemiología de los factores de riesgo en Colombia. Biomédica. 2008;28:480-96. https://doi.org/10.7705/biomedica.v28i4.54

8. Dáguer CF, Hernández-Tasco A. Héroes de a pie: voces y memorias de los detectives de la salud y la epidemiología de campo en Colombia. Bogotá: Instituto Nacional de Salud; 2019.

9. Idrovo AJ, Fernández-Niño JA, Bojórquez-Chapela I, Ruiz-Rodríguez M, Agudelo CA, Pacheco O, et al. Percepción de competencias en epidemiología en México y Colombia durante la epidemia de influenza $A\left(\mathrm{H}_{1} \mathrm{~N}_{1}\right)$ entre estudiantes de salud pública. Rev Panam Salud Pública. 2011;30:361-9.

10. Idrovo AJ, Fernández-Niño JA, Bojórquez-Chapela I, Moreno-Montoya J. Performance of public health surveillance systems during the influenza $A\left(\mathrm{H}_{1} \mathrm{~N}_{1}\right)$ pandemic in the Americas: Testing a new method based on Benford's law. Epidemiol Infect. 2011;139:1827-34 http://doi.org/10.1017/S095026881100015X

11. Benford F. The law of anomalous numbers. Proceedings of the American Philosophical Society. 1938;78:551-72.

12. Hill TP. The first digit phenomenon. American Scientist. 1998;86:358-63.

13. Formann AK. The Newcomb-Benford law in its relation to some common distributions. PLoS One. 2010;5:e10541. http://doi.org/10.1371/journal.pone.0010541

14. Durtschi C, Hillison W, Pacini C. The effective use of Benford's law to assist in detecting fraud in accounting data. J Forensic Account. 2004;5:17-34.

15. Lacasa L. Newcomb-Benford law helps customs officers to detect fraud in international trade. Proc Natl Acad Sci USA. 2019;116:11-3. http://doi.org/10.1073/pnas.1819470116

16. Gómez-Camponovo M, Moreno J, Idrovo ÁJ, Páez M, Achkar M. Monitoring the Paraguayan epidemiological dengue surveillance system (2009-2011) using Benford's law. Biomédica. 2016;36:583-92. http://doi.org/10.7705/biomedica.v36i4.2731

17. Manrique-Hernández EF, Fernández-Niño JA, Idrovo AJ. Global performance of epidemiologic surveillance of Zika virus: Rapid assessment of an ongoing epidemic. Public Health. 2017;143:14-6. http://doi.org/10.1007/s10900-016-0177-7

18. Idrovo AJ, Manrique-Hernández EF. Data quality of Chinese surveillance of COVID-19: Objective analysis based on WHO's situation reports. Asia Pac J Public Health. 2020. http://doi.org/10.1177/1010539520927265

19. Moreno-Montoya J. Benford's law with small sample sizes: A new exact test useful in health sciences during epidemics. Rev Salud UIS. 2020;52:153-5. http://dx.doi.org/10.18273/revsal.v52n2-2020010

20. Kuiper $\mathrm{NH}$. Tests concerning random points on a circle. Proceedings Koninklijke Nederlandse Akademie van Wetenschappen (Series A). 1962;63:38-47.

21. Gardy JL, Loman NJ. Towards a genomics-informed, real-time, global pathogen surveillance system. Nat Rev Genet. 2018;19:9-20. http://doi.org/10.1038/nrg.2017.88

22. Pacheco O, Beltrán M, Nelson CA, Valencia D, Tolosa N, Farr SL, et al. Zika virus disease in Colombia - Preliminary Report. N Engl J Med. 2016. http://doi.org/10.1056/NEJMoa1604037

23. Dong E, Du H, Gardner L. An interactive web-based dashboard to track COVID-19 in real time. Lancet Infect Dis. 2020. http://doi.org/10.1016/S1473-3099(20)30120

24. German RR, Lee LM, Horan JM, Milstein RL, Pertowski CA, Waller MN, et al. Updated guidelines for evaluating public health surveillance systems: Recommendations from the Guidelines Working Group. MMWR. 2001;50:1-35. 